\title{
Review Article \\ Clinical Cancer Therapy by NK Cells via Antibody-Dependent Cell-Mediated Cytotoxicity
}

\author{
Kory L. Alderson ${ }^{1,2}$ and Paul M. Sondel ${ }^{1,2,3}$ \\ ${ }^{1}$ Department of Human Oncology, University of Wisconsin, Madison, WI 53705, USA \\ ${ }^{2}$ Department of Pediatrics, University of Wisconsin, Madison, WI 53705, USA \\ ${ }^{3}$ Paul P. Carbone Comprehensive Cancer Center, University of Wisconsin, Madison, WI 53792-6164, USA
}

Correspondence should be addressed to Paul M. Sondel, pmsondel@humonc.wisc.edu

Received 1 February 2011; Accepted 16 March 2011

Academic Editor: Roberto Biassoni

Copyright (C) 2011 K. L. Alderson and P. M. Sondel. This is an open access article distributed under the Creative Commons Attribution License, which permits unrestricted use, distribution, and reproduction in any medium, provided the original work is properly cited.

\begin{abstract}
Natural killer (NK) cells are powerful effector cells that can be directed to eliminate tumor cells through tumor-targeted monoclonal antibodies (mAbs). Some tumor-targeted mAbs have been successfully applied in the clinic and are included in the standard of care for certain malignancies. Strategies to augment the antitumor response by NK cells have led to an increased understanding of how to improve their effector responses. Next-generation reagents, such as molecularly modified mAbs and mAbcytokine fusion proteins (immunocytokines, ICs) designed to augment NK-mediated killing, are showing promise in preclinical and some clinical settings. Continued research into the antitumor effects induced by NK cells and tumor-targeted mAbs suggests that additional intrinsic and extrinsic factors may influence the antitumor response. Therefore more research is needed that focuses on evaluating which NK cell and tumor criteria are best predictive of a clinical response and which combination immunotherapy regimens to pursue for distinct clinical settings.
\end{abstract}

\section{Introduction}

Natural killer (NK) cells are innate immune effector cells capable of recognizing and destroying virally infected and neoplastic cells. The importance of NK cell-mediated immunosurveillance in the control of tumor growth has been evaluated in NK cell-deficient mouse models with limited information in humans. Humans with NK cell deficiencies are plagued with persistent acute viral infections, especially herpes simplex virus [1]. However, mouse models with defects in NK cell effector function clearly demonstrate an increased susceptibility to neoplastic disease as they age [2].

NK cell effector functions can be exploited for the treatment of some tumors through their ability to mediate antibody-dependent cellular cytotoxicity (ADCC). The NK cell Fc receptor, CD16 (Fc $\gamma$ RIIIa), contains an immunotyrosine-activating motif (ITAM) in the cytoplasmic domain. NK cell recognition of an antibody-coated target cell results in rapid NK cell activation and degranulation
[3]. mAbs that specifically target tumor cells take advantage of the ADCC effector pathway to tip the balance of an interrogating $\mathrm{NK}$ cell in the favor of the activating receptors resulting in tumor cell destruction and an anti-tumor immune response [4].

Tumor-targeted mAbs that initiate NK cell ADCC have been used clinically. Antibodies targeting CD20, Her2/neu, epidermal growth factor receptor (EGFR), and disaloganglioside (GD2) are examples of clinically successful antibodies whose mechanisms include NK cellmediated ADCC [5-9]. GD2 is overexpressed on tumors of neuroectodermal origin, such as neuroblastoma and melanoma, and minimally expressed in normal tissues making it a good target for tumor-specific mAb. Anti-GD2 mAbs work through NK cell-mediated ADCC and have demonstrated clinical benefit for children with neuroblastoma [10]. In this paper, we will use examples from mAbs targeting GD2 and other tumor antigens to discuss antibody-facilitated NK cell-mediated cancer immunotherapy strategies. 


\section{NK Cell Responses to Tumor-Specific mAbs}

There are numerous $\mathrm{Fc}$ receptors for $\operatorname{IgG}(\mathrm{Fc} \gamma \mathrm{R})$ that are widely expressed on immune cells. The $\mathrm{Fc} \gamma \mathrm{R}$ family consists of four classes of receptors, Fc $\gamma$ RI, Fc $\gamma$ RII, Fc $\gamma$ RIII, and $\mathrm{Fc} \gamma \mathrm{RIV}$, that have been identified in both mice and humans. There are significant similarities in the functions of the $\mathrm{F} c \gamma \mathrm{R}$ receptors between mice and humans, but there is limited homology in receptors themselves [11]. To date, only one inhibitory $\mathrm{F} c \gamma \mathrm{R}, \mathrm{Fc} \gamma \mathrm{RIIb}$, has been identified and is the only receptor to have complete homology between mice and humans [11]. Fc $\gamma$ Rs can be found on virtually all hematopoietic cells except $\mathrm{T}$ cells; in most cases, cells coexpress activating and inhibitory $\mathrm{F} c \gamma \mathrm{R}$, allowing for the balance between activating and inhibitory receptors to dictate their response [11]. NK cells are an exception to this rule and express only the activating Fc $\gamma$ RIIIa. NK cells do not express the inhibitory Fc $\gamma$ RIIb.

Because of their rapid and unopposed responses to $\mathrm{mAb}$, NK cells play a major role in the anti-tumor response elicited by tumor-specific mAbs. Early studies demonstrated that NK cells are the primary mediators of the immune response elicited by tumor-targeted mAbs. However, recent data show that granulocytes may also play a significant role in antitumor responses generated by tumor-targeted antibodies $[11,12]$. NK cells are important effectors in the mAbdriven immune response to tumors, and data continue to accumulate on their importance [13]. Multiple clinically successful mAbs utilize NK-mediated ADCC as a mechanism of action. Rituximab (anti-CD20), Herceptin (anti-Her2/neu), Cetuximab (anti-EGFR), and the anti-GD2-mAbs 3F8 and ch14.18 are examples of tumor-specific mAbs whose clinical activity can be attributed, at least in part, to NK cells.

\section{Augmentation of mAb Responses through the Activation of NK Cells}

Various strategies of NK cell activation alongside antibody administration have been evaluated. NK cell activation can occur through a variety of stimuli including cytokine administration, toll-like receptor (TLR) agonists, or agonist antibodies directed toward activating receptors on NK cells. The coadministration of an NK-activating cytokine, interleukin-2 (IL-2), enhances the anti-tumor activity of NK cells $[14,15]$. The TLR9 agonist CpG can activate numerous innate immune effectors, including NK cells [16]. Combination of CpG with Rituximab increases NKmediated ADCC in vitro and anti-tumor responses in a mouse model of CD20-expressing tumors [17].

Further activation of NK cells through numerous receptors including $4-1 \mathrm{BB}$ or the $\mathrm{Fc}$ receptor $\mathrm{Fc} \gamma \mathrm{RIII}$ increases ADCC activity [18]. Antibodies with $\mathrm{Fc}$ regions that have higher affinity for Fc $\gamma$ RIIIa are better at activating NK cells while simultaneously initiating additional NK effector pathways [19]. High-affinity Fc-antibodies can be used at lower concentrations than traditional antibodies and maintain anti-tumor activity [19]. Antibodies with higher affinity for Fc $\gamma$ RIIIa may be beneficial in a clinical setting by reducing the amount of antibody necessary to produce an antitumor response and therefore reduce $\mathrm{mAb}$-related toxicities [20]. 4-1BB (CD137) is an activating receptor on the surface of NK cells [21]. The activation of NK cells with an agonistic antibody to $4-1 \mathrm{BB}$ has recently been described in a mouse model of B-cell lymphoma [18]. 4$1 \mathrm{BB}$ is increased on CD56 ${ }^{\mathrm{dim}}$ effector NK cells after CD16mediated activation. Activation of NK cells with a $4-1 \mathrm{BB}$ agonist antibody between Rituximab courses in vivo led to complete regression of subcutaneous murine lymphoma tumors by NK cells [18].

The combination of mAb therapy with cytokines is another strategy used to increase their activity. Combination of Herceptin with interleukin-12 (IL-12), an important cytokine to $\mathrm{NK}$ cell responsiveness and IFN $\gamma$ production, increases the response of NK cells to Her2-expressing breast tumor cells in a mouse model of breast cancer [22]. Clinical development of this concept is underway [23]. Augmentation of NK cell responses by the addition of exogenous IL-2 has been extensively demonstrated to increase the anti-tumor response of antibody therapy. IL-2-activated lymphokineactivated killer (LAK) cells have increased ADCC activity against mAb-coated tumor cells $[14,15,24]$. IL-2 lowers the required amount of antibody necessary for NK cells to effectively lyse antibody-coated tumor targets [25]. Increased NK cell effector function after IL-2 activation is true of NK cells isolated from humans, mice, and dogs [26, 27].

\section{Altered mAbs That Increase NK Cell Effector Functions}

Following the production of the initial 14.18 murine antiGD2 mAb, several molecular modifications have resulted in 2nd and 3rd generation reagents, designed to have improved function. First, there was the class switch to murine IgG2a to augment ADCC (creating the 14.G2a mAb). This was followed the by creation of a chimeric antibody (ch14.18), a humanized antibody (hu14.18), and multiple altered hu14.18 antibodies to enhance the anti-tumor response [10]. Humanized anti-GD2 mAb hu14.18K322A (K322A) is a new-generation anti-GD2 $\mathrm{mAb}$ that has been designed to stimulate NK cell effector mechanisms and simultaneously reduce some of the toxicities associated with anti-GD2 therapy [5]. hu14.18K322A has two key differences from its hu14.18 parent. First, hu14.18K322A was produced in a rat hybridoma line, YB2/0. YB2/0 cells have low fucosyltrasferase activity and therefore produce antibodies with fewer fucose side chains on the Fc portion. IgG antibodies that have low or absent fucose side chains are more effective at eliciting ADCC $[28,29]$. Second, hu14.18K322A has a point mutation at the 322 position resulting in the replacement of lysine 322 with an alanine. This specific mutation reduces the ability of hu14.18K322A to activate complement compared to its anti-GD2 relatives [30]. Allodynia, the major clinical toxicity associated with anti-GD2 therapy, is likely the result of complement fixation. Therefore, hu14.18K322A is designed to retain or potentially enhance NK-mediated anti-tumor responses while reducing the antibody's toxicity [30]. 
Immunocytokines (ICs) are antibodies with linked cytokines at the Fc terminal end. The anti-GD2 IC hu14.18IL-2 is a humanized mAb with two functional interleukin2 proteins at the $\mathrm{Fc}$ terminal end $[31,32]$. ICs may have certain advantages over traditional mAbs [33]. In several preclinical models, using 3 different ICs, the IC provided far greater antitumor effects than the same amount of the naked $\mathrm{mAb}$ infused with the same amount of IL2 (but infused simultaneously as separate molecules rather than as the IC fusion protein). This may be because ICs transport cytokine to the site of tumor and can support an ongoing local anti-tumor immune response [34, 35]. Direct delivery of IC into the tumor itself elicits a more potent local effect. Intratumoral injection of IC in tumor-bearing mice induces better antitumor responses than systemic administration. This effect can be attributed to its activating effects on intratumoral NK cells [34].

One advantage of using IC is its effect on the formation of an immune synapse between the Ab-coated tumor cell and the NK cell. Recent data from our laboratory suggest that NK recognition of an IC involves not only the Fc receptor, but also IL2 receptors $[36,37]$. The involvement of the IL2R increases IC-facilitated conjugate formation between NK cells and tumor cells (Figure 1) [36, 37]. Furthermore, IC may facilitate NK: tumor cell conjugation in the absence of $\mathrm{Fc}$ receptors. Using an $\mathrm{NK}$ cell line with minimal, if any, expression of Fc $\gamma$ RIIIa, we recently demonstrated that the IL2R $\alpha$ chain plays an important role in NK: tumor cell conjugation [36]. The association of NK cells with IC-coated tumor cell results in the formation of an activated immune synapse (AIS), defined by the localization of LFA-1and CD2 [37]. AIS formation facilitated by an IC is hallmarked by clustering of NK cell CD25 into the synapse and can also be abrogated by CD25 blockade [37]. The potential benefit of IL-2 containing ICs in activating and assisting NK cells in tumor cell destruction is a relatively new research area for clinical NK-mediated tumor immunotherapy.

\section{Recent Clinical Results with Anti-GD2 mAb- Induced ADCC}

High-risk childhood neuroblastoma remains a disease that has not shown major improvements in cure rates over the past 2 decades [38]. Preclinical and early clinical work suggested that anti-GD2-based mAb therapy would be most effective if given in the setting of minimal residual disease, and in combination with cytokines that augment ADCC. A large randomized study was conducted by the Children's Oncology Group (COG) to test these concepts. Following initial response to combined agent chemotherapy, surgery, ablative chemotherapy, and autologous hematopoietic stem cell transplant, children received isotretinoin and were randomized to receive immunotherapy (the ch14.18 chimeric mAb + IL2 + GM-CSF) or no immunotherapy. Two-hundred twenty-six children were randomized, and the group receiving immunotherapy showed a 2 -year eventfree survival of $66 \%$ versus $46 \%$ for the no-immunotherapy group $(P=.012)$ [39]. In the USA, through the COG, this immunotherapy regimen has thus become the "standard of care" maintenance treatment for high-risk patients that have responded to their initial therapy.

The next-generation immunotherapy approach has involved the hu14.18-IL2 IC. Our hypothesis, based on our preclinical data [27], was that the IC approach would work best for children with smaller amounts of refractory/relapsed neuroblastoma. A recent phase II COG study in children with relapsed or refractory neuroblastoma showed that 7 of 24 patients with "nonbulky" disease showed evidence of antitumor activity, while 0 of 13 with bulky disease had evidence of antitumor activity [40]. The results of this study are consistent with the hypothesis of better activity for nonbulky disease $(P=.03)$. We are continuing to develop this agent and are hoping that a future large COG trial will enable us to test this genetically engineered molecule for children in remission in order to prevent relapse, as was done for the separate ch14.18 mAb with GM-CSF and IL2 regimen [39].

\section{Fc Receptor Polymorphisms and NK Responses to mAbs and ICs}

Two allelic polymorphisms have been identified in human Fc $\gamma$ RIIIa at position 158. The aa at this location in the receptor interacts with the hinge region of IgG antibodies and affects the magnitude of response at subsaturating concentrations of $\operatorname{IgG}$ [41]. The one aa difference of either a phenylalanine (f) or a valine (v) in Fc $\gamma$ RIIIa may have implications for mAb therapy [42]. NK cells containing a valine at position 158 have a higher affinity for IgG. NK cells isolated from individuals bearing this receptor contain more cytophilic IgG when examined directly ex vivo [42]. Fc $\gamma$ RIIIa $^{158 v}$ is associated with a less favorable prognosis for autoimmune sufferers [43]. Fc $\gamma$ RIIIa $^{158 v}$ NK cells are more sensitive to activation and have a higher calcium influx and more rapid induction of activation-induced cell death (AICD) when stimulated [44]. A more sensitive activating receptor that produces a stronger intracellular response, such as $\mathrm{Ca}^{2+}$ influx, may support a more productive immune synapse by localizing granules faster to the centrosome, as has been shown with T-cell responses to TCR stimulation [45]. Faster granule localization results in more granules loaded into the synapse for target cell destruction [45]. Therefore, NK cells with Fc $\gamma$ RIIIa $^{158 v}$ receptors may have a twofold advantage in the setting of mAb-mediated cancer immunotherapy: (1) enhanced ability to recognize and bind to tumor cells coated with mAb molecules and (2) the release of more granules for each tumor cell they encounter.

Patients with an Fc $y$ RIIIa $^{158 v}$ genotype respond better to therapy that utilizes an ADCC-mediating $\mathrm{mAb}$ as a mechanism of action. Studies using patient samples during treatment with Herceptin, Rituximab, and Cetuximab have correlated an Fc $\gamma$ RIIIa $^{158 v}$ receptor genotype with better response to therapy [46-48]. The response of Fc $\gamma$ RIIIa $^{158 \mathrm{v}}$ NK cells in an in vitro assay of ADCC against antibodycoated tumor cells can be used as a predictor for patient response to therapy [49]. The role of Fc receptor polymorphisms on the response to immunocytokine in comparison 


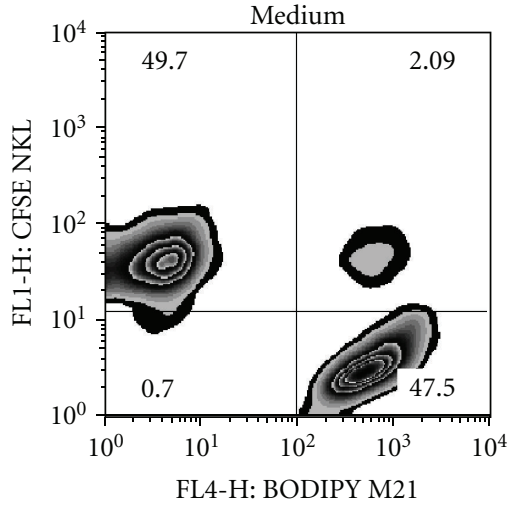

(a)

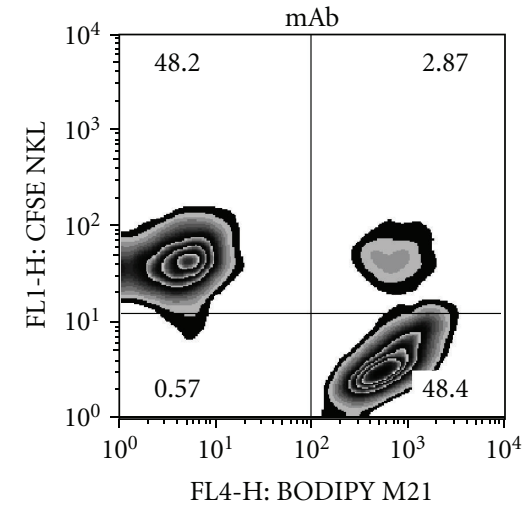

(b)

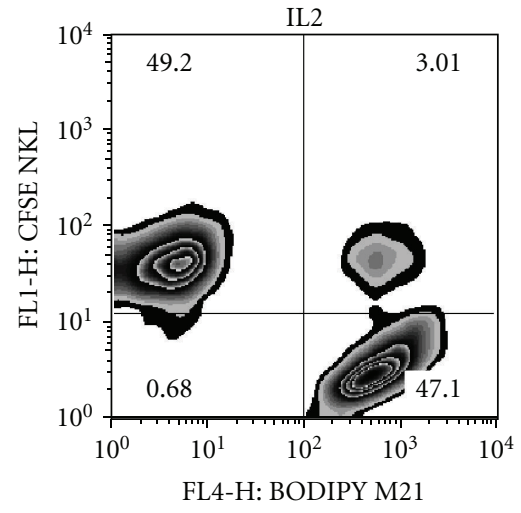

(c)

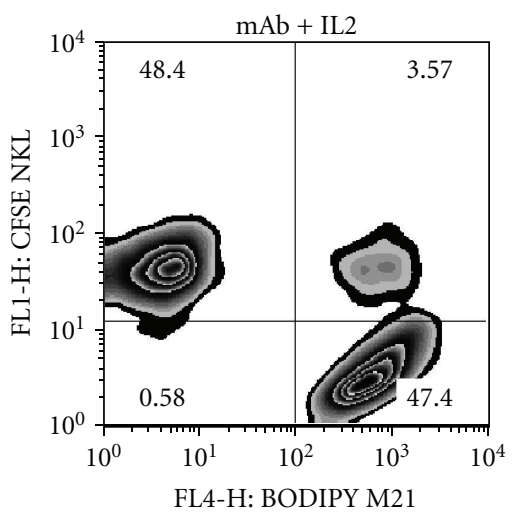

(d)

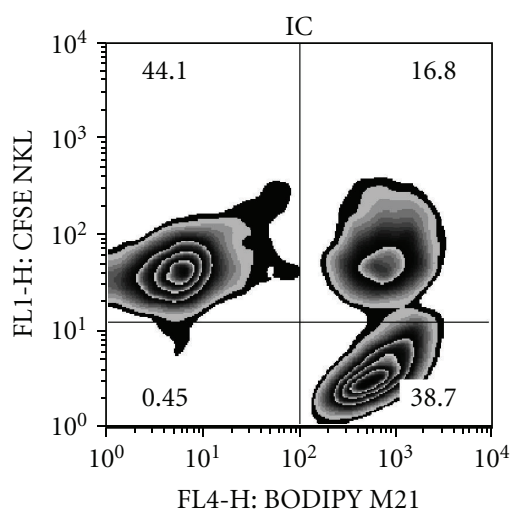

(e)

FIgURE 1: Immunocytokine facilitates adhesion of NK cells to tumor cells. This figure, adapted from Buhtoiarov et al. [36], shows the adhesion of M21 human melanoma tumor cells to NKL human NK cells after coincubation for 30 minutes. Tumor cells were prelabeled with CFSE, and NK cells were prelabeled with BODIPY and coincubated in medium alone, with hu14.18 (mAb) alone, soluble IL2, hu14.18 + soluble IL2, or hu14.18-IL2 immunocytokine (IC). The percentage of cells that had formed conjugates was defined as double positive cells as determined by flow cytometry.

with $\mathrm{mAb}$ remains to be answered. A recent study from our laboratory [12] evaluated patient samples from our COG phase II anti-GD2 IC trial for Fc $\gamma$ RIIIa genotypes [40]. This study only had two patients with an FcyRIIIa ${ }^{158 \mathrm{v} / \mathrm{v}}$ genotype and was therefore inconclusive. The identification of $\mathrm{Fc} \gamma \mathrm{R}$ polymorphisms that affect clinical responses to $\mathrm{mAb}$ therapy may suggest a new criterion for more tailored patient selection for $\mathrm{mAb}$ therapy. The importance of Fc $\gamma$ RIIIa polymorphisms in IC-induced anti-tumor responses will be of considerable interest because of the involvement of the IL2R.

\section{KIRs and the Response to mAbs and ICs}

An important factor in NK-mediated therapy is the intrinsic ability for NK cells to respond to stimuli. The licensing hypothesis of NK cell function was developed after the observation that NK cells from mice lacking MHC-I, an important NK inhibitory ligand, respond poorly [50]. The intricate system of killer cell immunoglobulin-like receptors (KIRs) in humans and Ly-49 receptors in mice recognizes normally expressed MHC-I antigens on neighboring cells and inhibit NK cell effector functions [51]. According to the licensing hypothesis, an NK cell that does not encounter a ligand for one of its KIR or Ly49 inhibitory receptors during development is functionally deficient [50]. However, KIR and Ly49 genes are inherited on different chromosomes from MHC, and therefore many individuals and mouse strains have at least one KIR or Ly49 that lacks a corresponding ligand [51]. These individuals and mouse strains have at least one population of functionally deficient NK cells [50], reflecting the NK population that contains the KIR receptor corresponding to the KIR/KIR-L mismatch. Clinical studies evaluating KIR/KIR-L matching have estimated that $\sim 60 \%$ of people have at least one KIR for which they lack a corresponding receptor and are therefore KIR/KIR-L mismatched for at least one locus [51].

Some preclinical studies have challenged the importance of licensing in certain models. Orr et al. evaluated the response of licensed versus unlicensed NK cells in response to murine cytomegalovirus (MCMV). $\mathrm{C} 57 \mathrm{Bl} / 6$ mice have a "licensed" subset of NK cells expressing Ly49C/I, the receptor for $\mathrm{H}_{2}{ }^{\mathrm{b}}$ and "unlicensed" NK cells expressing an 
activating Ly49H. After infection of B6 mice with MCMV, a virus for which NK cell function is necessary for control, $\mathrm{LY} 49 \mathrm{C} / \mathrm{I}^{\text {neg }} \mathrm{Ly} 49 \mathrm{H}^{+}$("unlicensed") NK cells were able to respond to infection [52]. This paper elegantly demonstrated that unlicensed NK cells still maintain some functionality to activating signals, although their effector responses are still less powerful than that of licensed NK cells.

New data suggest that "licensing" may not be a process restricted to NK cell development but is actually a continuous process that even mature NK cells use to respond to their environment $[53,54]$. Recently, two separate reports compared adoptive transfer studies between wild type (WT) and $\beta 2 \mathrm{~m}$ knockout (MHC-I deficient) mice to show that mature NK cells become hyporesponsive when they are put into an environment lacking $\mathrm{MHC}$ and vice versa $[53,54]$. In these studies, only NK cells expressing an inhibitory Ly49 for which there was a cognate MHC-I ligand present could respond in the new surroundings. These studies suggest that "licensing" is a continuous (rather than absolute) mechanism that NK cells use to judge and appropriately respond to a changing environment.

While studies using murine models have been valuable in dissecting the potential factors affecting NK cell responsiveness, some observations of better anti-tumor responses by patients that are self-KIR/KIR-L mismatched have been made at multiple institutions $[12,55,56]$. Two studies evaluated anti-tumor responses in pediatric cancer patients following autologous stem cell transplant $[55,56]$. These two independent studies both observed an association between disease-free survival and autologous KIR/KIR-L mismatch. Our laboratory recently evaluated the KIR/KIR-L status [12] of neuroblastoma patient samples from our COG phase II study of the anti-GD2-IC, hu14.18-IL2 [40]. In this study, we observed a better response to IC in patients that were KIR/KIR-L mismatched. These data suggest that the "unlicensed" NK cells in these patients were still involved in mediating the observed antitumor effect after IC treatment, consistent with some retained NK function by "unlicenced" NK cells. Previous reports have suggested that KIR-deficient NK cells have impaired responses to CD16-mediated stimulation [57]. In this study using NK cells from healthy donors, fewer KIR-deficient CD56 ${ }^{\text {dim }}$ NK cells produced IFN $\gamma$ or upregulated CD107a in response to either antibody-coated tumor cells or plate bound anti-CD16. However, our study using patient samples [12] and evaluating response to IC rather than naked $\mathrm{mAb}$ warrants a closer examination of $\mathrm{NK}$ cell "licensing" in the context of IC-mediated ADCC.

\section{Conclusions}

In the relatively short time since the first description of NK cells, just over 35 years ago [58], we have learned a great deal about their function. This knowledge has allowed us to design therapeutic strategies that utilize the powerful effector mechanisms of NK cells for multiple malignancies $[6-8,40]$. Our understanding of NK cells, NK cell effector responses, and the signals that drive them, continues to expand. NK cells are capable of eliminating tumor cells coated with an
IgG antibody in vitro and in some patients, and activation of NK cells with cytokines such as IL2 increases the anti-tumor effect $[14,15,24,46]$. This approach has led to successful therapeutic mAbs for certain tumors but still do not elicit a response in all patients [38].

Our continued understanding of the factors that affect the response to Ab-coated tumor cells in the tumor environment is important for the creation of the nextgeneration $\mathrm{mAbs}$ and therapeutic strategies. Important intrinsic factors that affect $\mathrm{NK}$ cell responsiveness to $\mathrm{Ab}-$ coated tumor cells include the expressed variants of Fc $\gamma$ RIIIa and the intrinsic ability of individual NK cell subsets to respond to stimuli due to their "licensing" status $[12,50]$. Separate factors include the mass of tumor present when immunotherapy is given, the activation state of the effector cells mediating ADCC, and potential other receptor-ligand interactions that influence the synapse formed between NK and tumor cells. Next-generation immunocytokines may have a functional advantage over traditional mAbs in activation of certain NK cell subsets because of the contribution of cytokine receptors [33, 36, 37].

\section{Acknowledgments}

The authors thank Tony Koehn for his help with editing the paper. This work was supported by National Institutes of Health Grants nos. CA032685, CA87025, CA14520, and GM067386, and grants from the Midwest Athletes for Childhood Cancer Fund, the Crawdaddy Foundation, The Evan Dunbar Foundation, the UW-Cure Kids Cancer Coalition, Abbie's Fund, and the Super Jake Foundation.

\section{References}

[1] J. S. Orange, "Human natural killer cell deficiencies and susceptibility to infection," Microbes and Infection, vol. 4, no. 15, pp. 1545-1558, 2002.

[2] J. A. Trapani and I. Voskoboinik, "Infective, neoplastic, and homeostatic sequelae of the loss of perforin function in humans," Advances in experimental medicine and biology, vol. 601, pp. 235-242, 2007.

[3] J. V. Ravetch and S. Bolland, "IgG Fc receptors," Annual Review of Immunology, vol. 19, pp. 275-290, 2001.

[4] P. M. Sondel and J. A. Hank, "Antibody-directed, effector cellmediated tumor destruction," Hematology/Oncology Clinics of North America, vol. 15, no. 4, pp. 703-721, 2001.

[5] F. Navid, V. M. Santana, and R. C. Barfield, "Anti-GD2 antibody therapy for GD2-expressing tumors," Current Cancer Drug Targets, vol. 10, no. 2, pp. 200-209, 2010.

[6] K. P. Garnock-Jones, G. M. Keating, and L. J. Scott, "Trastuzumab: a review of its use as adjuvant treatment in human epidermal growth factor receptor 2 (HER2)-positive early breast cancer," Drugs, vol. 70, no. 2, pp. 215-239, 2010.

[7] J. García-Foncillas and E. Díaz-Rubio, "Progress in metastatic colorectal cancer: growing role of cetuximab to optimize clinical outcome," Clinical and Translational Oncology, vol. 12, no. 8, pp. 533-542, 2010.

[8] M. C. Winter and B. W. Hancock, "Ten years of rituximab in NHL," Expert Opinion on Drug Safety, vol. 8, no. 2, pp. 223$235,2009$. 
[9] M. R. Albertini, J. A. Hank, and P. M. Sondel, "Native and genetically engineered anti-disialoganglioside monoclonal antibody treatment of melanoma," Cancer Chemotherapy and Biological Response Modifiers, vol. 22, pp. 789-797, 2005.

[10] R. K. Yang and P. M. Sondel, "Anti-GD2 strategy in the treatment of neuroblastoma," Drugs Future, vol. 35, no. 8, p. 665,2010 .

[11] F. Nimmerjahn and J. V. Ravetch, "Fcy receptors as regulators of immune responses," Nature Reviews Immunology, vol. 8, no. 1, pp. 34-47, 2008.

[12] D. C. Delgado, J. A. Hank, J. Kolesar et al., "Genotypes of NK cell KIR receptors, their ligands, and Fcy receptors in the response of neuroblastoma patients to Hu14.18-IL2 immunotherapy," Cancer Research, vol. 70, no. 23, pp. 9554 9561, 2010.

[13] A. Beano, E. Signorino, A. Evangelista et al., "Correlation between NK function and response to trastuzumab in metastatic breast cancer patients," Journal of Translational Medicine, vol. 6, article no. 25, 2008.

[14] J. A. Hank, G. Weil-Hillman, J. E. Surfus, J. A. Sosman, and P. M. Sondel, "Addition of interleukin-2 in vitro augments detection of lymphokine-activated killer activity generated in vivo," Cancer Immunology Immunotherapy, vol. 31, no. 1, pp. 53-59, 1990.

[15] J. R. Ortaldo, C. Woodhouse, and A. C. Morgan, "Analysis of effector cells in human antibody-dependent cellular cytotoxicity with murine monoclonal antibodies," Journal of Immunology, vol. 138, no. 10, pp. 3566-3572, 1987.

[16] H. H. Van Ojik, L. Bevaart, C. E. Dahle et al., "CpG-A and B oligodeoxynucleotides enhance the efficacy of antibody therapy by activating different effector cell populations," Cancer Research, vol. 63, no. 17, pp. 5595-5600, 2003.

[17] D. J. Betting, R. E. Yamada, K. Kafi, J. Said, N. Van Rooijen, and J. M. Timmerman, "Intratumoral but not systemic delivery of $\mathrm{CpG}$ oligodeoxynucleotide augments the efficacy of anti-CD20 monoclonal antibody therapy against B Cell lymphoma," Journal of Immunotherapy, vol. 32, no. 6, pp. 622631, 2009.

[18] H. E. Kohrt, R. Houot, M. J. Goldstein et al., "CD137 stimulation enhances the antilymphoma activity of anti-CD20 antibodies," Blood, vol. 117, no. 8, pp. 2423-2432, 2011.

[19] J. A. Bowles, S. Y. Wang, B. K. Link et al., "Anti-CD20 monoclonal antibody with enhanced affinity for CD16 activates NK cells at lower concentrations and more effectively than rituximab," Blood, vol. 108, no. 8, pp. 2648-2654, 2006.

[20] L. M. Weiner, R. Surana, and S. Wang, "Monoclonal antibodies: versatile platforms for cancer immunotherapy," Nature Reviews Immunology, vol. 10, no. 5, pp. 317-327, 2010.

[21] W. Lin, C. J. Voskens, X. Zhang et al., "Fc-dependent expression of CD137 on human NK cells: insights into "agonistic" effects of anti-CD137 monoclonal antibodies," Blood, vol. 112, no. 3, pp. 699-707, 2008.

[22] R. Parihar, J. Dierksheide, Y. Hu, and W. E. Carson, "IL-12 enhances the natural killer cell cytokine response to Ab-coated tumor cells," Journal of Clinical Investigation, vol. 110, no. 7, pp. 983-992, 2002.

[23] R. Parihar, P. Nadella, A. Lewis et al., "A phase I study of interleukin 12 with trastuzumab in patients with human epidermal growth factor receptor-2-overexpressing malignancies: analysis of sustained interferon $\gamma$ production in a subset of patients," Clinical Cancer Research, vol. 10, no. 15, pp. 50275037, 2004.

[24] K. R. Schultz, J. P. Klarnet, D. J. Peace et al., "Monoclonal antibody therapy of murine lymphoma: enhanced efficacy by concurrent administration of interleukin 2 or lymphokineactivated killer cells," Cancer Research, vol. 50, no. 17, pp. 5421-5425, 1990.

[25] M. Watanabe, T. Kubota, M. Kitajima, and S. Hakomori, "Synergetic effect of interleukin-2 and cellular cytotoxicity against a novel tumor-associated carbohydrate antigen $\mathrm{Le}(\mathrm{a}) / \mathrm{Le}(\mathrm{a})$ (dimeric Le(a) mediated by monoclonal antibody NCC-ST421 in adoptive immunization using SCID mice," Cancer Immunology Immunotherapy, vol. 37, no. 4, pp. 245-250, 1993.

[26] S. C. Helfand, S. A. Soergel, R. L. Donner et al., "Potential to involve multiple effector cells with human recombinant interleukin-2 and antiganglioside monoclonal antibodies in a canine malignant melanoma immunotherapy model," Journal of Immunotherapy, vol. 16, no. 3, pp. 188-197, 1994.

[27] Z. C. Neal, J. C. Yang, A. L. Rakhmilevich et al., "Enhanced activity of hu14.18-IL2 immunocytokine against murine NXS2 neuroblastoma when combined with interleukin 2 therapy," Clinical Cancer Research, vol. 10, no. 14, pp. 48394847, 2004.

[28] S. Iida, R. Kuni-Kamochi, K. Mori et al., "Two mechanisms of the enhanced antibody-dependent cellular cytotoxicity (ADCC) efficacy of non-fucosylated therapeutic antibodies in human blood," BMC Cancer, vol. 9, article no. 58, 2009.

[29] T. Shinkawa, K. Nakamura, N. Yamane et al., "The absence of fucose but not the presence of galactose or bisecting $\mathrm{N}$ acetylglucosamine of human IgG1 complex-type oligosaccharides shows the critical role of enhancing antibody-dependent cellular cytotoxicity," Journal of Biological Chemistry, vol. 278, no. 5, pp. 3466-3473, 2003.

[30] L. S. Sorkin, M. Otto, W. M. Baldwin et al., "Anti-GD with an FC point mutation reduces complement fixation and decreases antibody-induced allodynia," Pain, vol. 149, no. 1, pp. 135-142, 2010.

[31] S. D. Gillies, D. Young, K. M. Lo, and S. Roberts, "Biological activity and in vivo clearance of antitumor antibody/cytokine fusion proteins," Bioconjugate Chemistry, vol. 4, no. 3, pp. 230$235,1993$.

[32] J. A. Hank, J. E. Surfus, J. Gan et al., "Activation of human effector cells by a tumor reactive recombinant antiganglioside GD interleukin-2 fusion protein (ch14.18-IL2)," Clinical Cancer Research, vol. 2, no. 12, pp. 1951-1959, 1996.

[33] B. H. Yamane, J. A. Hank, M. R. Albertini, and P. M. Sondel, "The development of antibody-IL-2 based immunotherapy with hu14.18-IL2 (EMD-273063) in melanoma and neuroblastoma," Expert Opinion on Investigational Drugs, vol. 18, no. 7, pp. 991-1000, 2009.

[34] E. E. Johnson, H. D. Lum, A. L. Rakhmilevich et al., "Intratumoral immunocytokine treatment results in enhanced antitumor effects," Cancer Immunology, Immunotherapy, vol. 57, no. 12, pp. 1891-1902, 2008.

[35] H. N. Lode, R. Xiang, N. M. Varki, C. S. Dolman, S. D. Gillies, and R. A. Reisfeld, "Targeted interleukin-2 therapy for spontaneous neuroblastoma metastases to bone marrow," Journal of the National Cancer Institute, vol. 89, no. 21, pp. 1586-1594, 1997.

[36] I. N. Buhtoiarov, Z. C. Neal, J. Gan et al., "Differential internalization of hu14.18-IL2 immunocytokine by NK and tumor cell: impact on conjugation, cytotoxicity, and targeting," Journal of Leukocyte Biology, vol. 89, no. 4, pp. 625-638, 2011.

[37] J. A. A. Gubbels, B. Gadbaw, I. N. Buhtoiarov et al., "Ab-IL2 fusion protein mediate NK cell immune synapse formation by polarizing CD25 to the target cell-effector cell interface," Cancer Immunology and Immunotherapy. Manuscript Submitted. 
[38] J. M. Maris, "Recent advances in neuroblastoma," New England Journal of Medicine, vol. 362, no. 23, pp. 2154-2211, 2010.

[39] A. L. Yu, A. L. Gilman, M. F. Ozkaynak et al., "Anti-GD2 antibody with GM-CSF, interleukin-2, and isotretinoin for neuroblastoma," New England Journal of Medicine, vol. 363, no. 14, pp. 1324-1334, 2010.

[40] S. Shusterman, W. B. London, S. D. Gillies et al., "Antitumor activity of Hu14.18-IL2 in patients with relapsed/refractory neuroblastoma: a Children's Oncology Group (COG) phase II study," Journal of Clinical Oncology, vol. 28, no. 33, pp. 4969 4975, 2010.

[41] S. Dall'Ozzo, S. Tartas, G. Paintaud et al., "Rituximabdependent cytotoxicity by natural killer cells: influence of FCGR3A polymorphism on the concentration-effect relationship," Cancer Research, vol. 64, no. 13, pp. 4664-4669, 2004.

[42] H. R. Koene, M. Kleijer, J. Algra, D. Roos, A. E. G. KR. Von Dem Borne, and M. De Haas, "Fc $\gamma$ RIIIa-158V/F polymorphism influences the binding of IgG by natural killer cell FC $\gamma$ RIIIa, independently of the FC $\gamma$ RIIIa-48L/R/H phenotype," Blood, vol. 90, no. 3, pp. 1109-1114, 1997.

[43] H. M. Dijstelbloem, R. H. M. Scheepers, W. W. Oost et al., "Fc $y$ receptor polymorphisms in Wegener's granulomatosis: risk factors for disease relapse," Arthritis and Rheumatism, vol. 42, no. 9, pp. 1823-1827, 1999.

[44] J. Wu, J. C. Edberg, P. B. Redecha et al., "A novel polymorphism of Fc $\gamma$ RIIIa (CD16) alters receptor function and predisposes to autoimmune disease," Journal of Clinical Investigation, vol. 100, no. 5, pp. 1059-1070, 1997.

[45] M. R. Jenkins, A. Tsun, J. C. Stinchcombe, and G. M. Griffiths, "The strength of T cell receptor signal controls the polarization of cytotoxic machinery to the immunological synapse," Immunity, vol. 31, no. 4, pp. 621-631, 2009.

[46] G. Cartron, L. Dacheux, G. Salles et al., "Therapeutic activity of humanized anti-CD20 monoclonal antibody and polymorphism in IgG Fc receptor FcyrIIIa gene," Blood, vol. 99, no. 3, pp. 754-758, 2002.

[47] A. Musolino, N. Naldi, B. Bortesi et al., "Immunoglobulin $\mathrm{g}$ fragment $\mathrm{c}$ receptor polymorphisms and clinical efficacy of trastuzumab-based therapy in patients with HER-2/neupositive metastatic breast cancer," Journal of Clinical Oncology, vol. 26, no. 11, pp. 1789-1796, 2008.

[48] WU. Zhang, M. Gordon, A. M. Schultheis et al., "FCGR2A and FCGR3A polymorphisms associated with clinical outcome of epidermal growth factor receptor-expressing metastatic colorectal cancer patients treated with single-agent cetuximab," Journal of Clinical Oncology, vol. 25, no. 24, pp. 3712-3718, 2007.

[49] W. K. Weng and R. Levy, "Two immunoglobulin G fragment $\mathrm{C}$ receptor polymorphisms independently predict response to rituximab in patients with follicular lymphoma," Journal of Clinical Oncology, vol. 21, no. 21, pp. 3940-3947, 2003.

[50] A. H. Jonsson and W. M. Yokoyama, "Natural killer cell tolerance licensing and other mechanisms," Advances in Immunology, vol. 101, pp. 27-79, 2009.

[51] C. Vilches and P. Parham, "KIR: diverse, rapidly evolving receptors of innate and adaptive immunity," Annual Review of Immunology, vol. 20, pp. 217-251, 2002.

[52] M. T. Orr, W. J. Murphy, and L. L. Lanier, "'Unlicensed' natural killer cells dominate the response to cytomegalovirus infection," Nature Immunology, vol. 11, no. 4, pp. 321-327, 2010.

[53] J. M. Elliott, J. A. Wahle, and W. M. Yokoyama, "MHC class Ideficient natural killer cells acquire a licensed phenotype after transfer into an MHC class I-sufficient environment," Journal of Experimental Medicine, vol. 207, no. 10, pp. 2073-2079, 2010.

[54] N. T. Joncker, N. Shifrin, F. Delebecque, and D. H. Raulet, "Mature natural killer cells reset their responsiveness when exposed to an altered MHC environment," Journal of Experimental Medicine, vol. 207, no. 10, pp. 2065-2072, 2010.

[55] W. Leung, R. Handgretinger, R. Iyengar, V. Turner, M. S. Holladay, and G. A. Hale, "Inhibitory KIR-HLA receptorligand mismatch in autologous haematopoietic stem cell transplantation for solid tumour and lymphoma," British Journal of Cancer, vol. 97, no. 4, pp. 539-542, 2007.

[56] J. M. Venstrom, J. Zheng, N. Noor et al., "KIR and HLA genotypes are associated with disease progression and survival following autologous hematopoietic stem cell transplantation for high-risk neuroblastoma," Clinical Cancer Research, vol. 15, no. 23, pp. 7330-7334, 2009.

[57] N. Anfossi, P. André, S. Guia et al., "Human NK cell education by inhibitory receptors for MHC class I," Immunity, vol. 25, no. 2, pp. 331-342, 2006.

[58] R. Kiessling, E. Klein, and H. Wigzell, “'Natural' killer cells in the mouse. I. Cytotoxic cells with specificity for mouse Moloney leukemia cells. Specificity and distribution according to genotype," European Journal of Immunology, vol. 5, no. 2, pp. 112-117, 1975. 


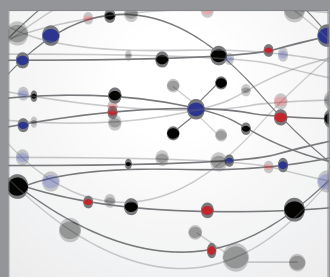

The Scientific World Journal
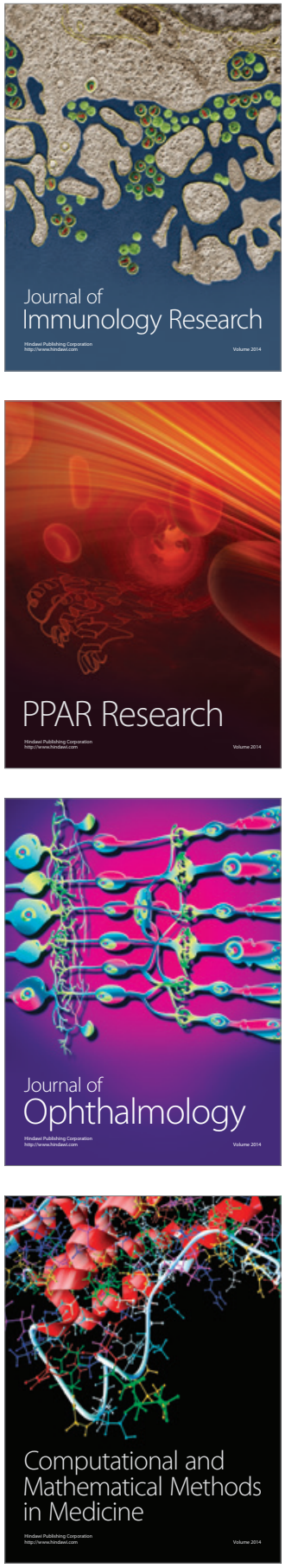

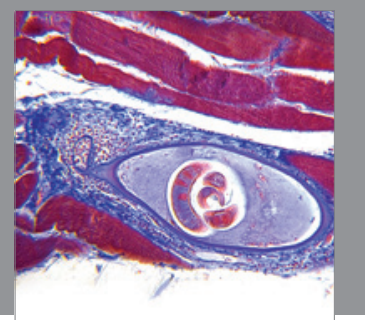

Gastroenterology

Research and Practice
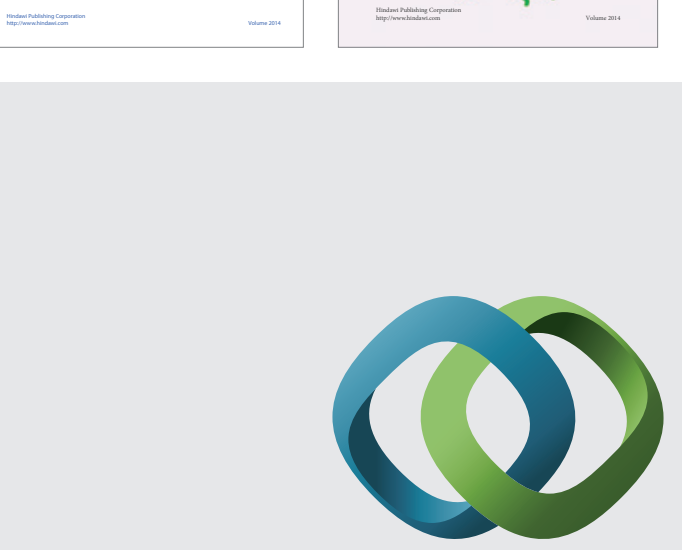

\section{Hindawi}

Submit your manuscripts at

http://www.hindawi.com
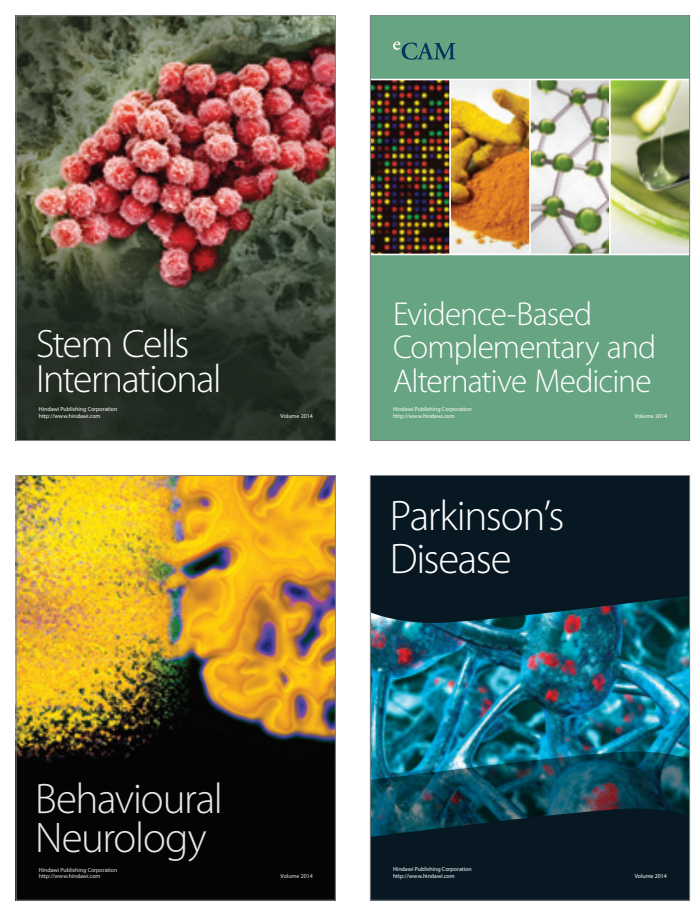

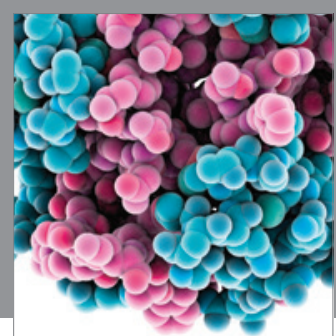

Journal of
Diabetes Research

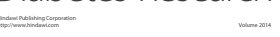

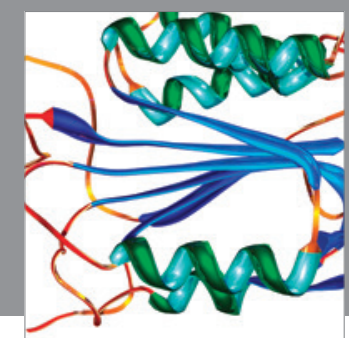

Disease Markers
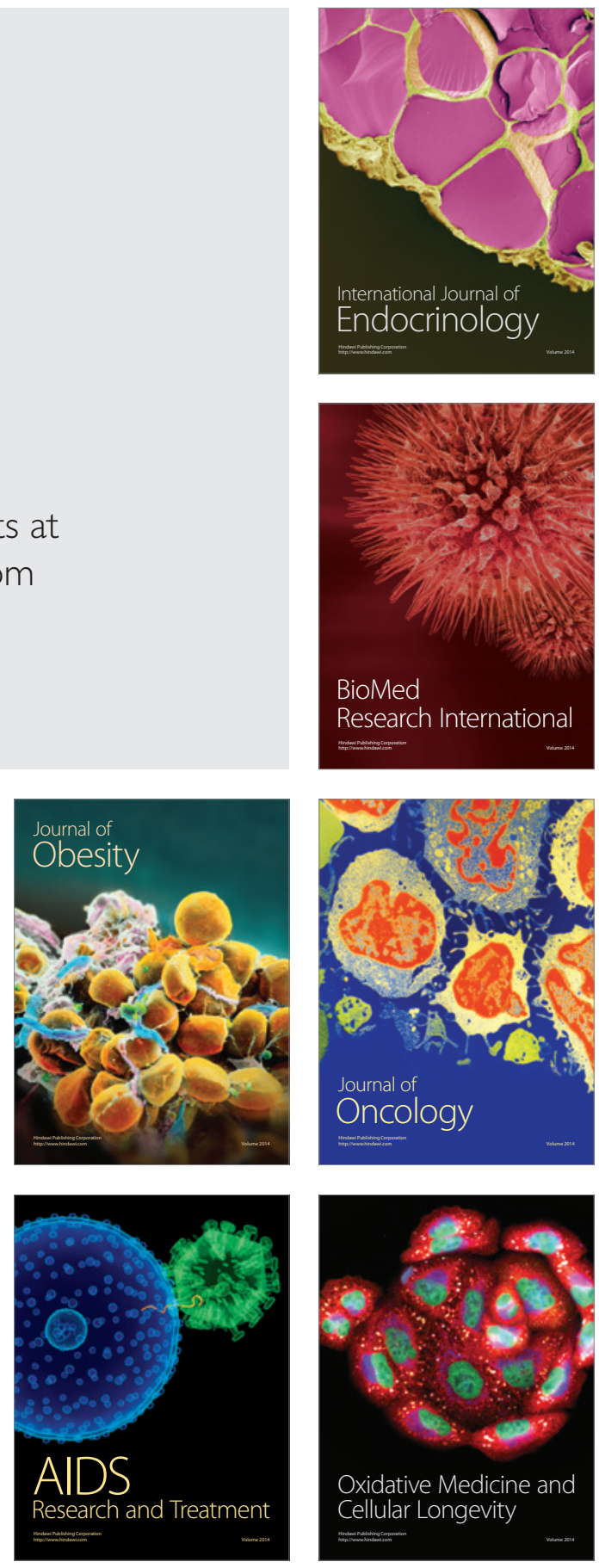